ESAIM: PROCEEDINGS, September 2007, Vol.21, 21-30

Gabriel Caloz \& Monique Dauge, Editors

\title{
ANALYSIS OF AN $\epsilon$-ARBITRAGE MODEL FOR INCOMPLETE MARKETS
}

\author{
Marie-Noelle Le RouX ${ }^{1}$ \\ Dedicated to Prof. Michel Crouzeix
}

\begin{abstract}
The optimal replication strategy for incomplete markets is obtained by solving a system of partial differential equations. In this paper, we study existence and uniqueness of the solution of this system and propose a numerical method to compute this optimal strategy.
\end{abstract}

Résumé. La stratégie optimale de réplication pour les marchés incomplets est obtenue par la résolution d'un système d'équations aux dérivées partielles. Dans cet article, on étudie l'existence et l'unicité de la solution de ce système et on propose une méthode numérique pour calculer cette stratégie optimale.

\section{INTRODUCTION}

Given a European derivative security with an arbitrary payoff function, we consider the optimal-replication problem: find a self-financing, dynamic portfolio strategy that most closely approximates the payoff function at maturity.

Under some hypotheses (complete markets), the payoff of a European option can be replicated exactly; it is the Black and Scholes model (1973) [2] .

But the conditions that guarantee dynamics spanning are nontrivial restrictions on market structures and price dynamics; hence there are situations in which exact replication is impossible (for example, if the volatility is stochastic). In [1], Bertsimas, Kogan and Lo propose a solution approach for the problem of replicating a derivative security in incomplete markets.

At time $\tau=0$, consider a portfolio of stocks and riskless bonds at a cost $V_{0}$ and denote by $\theta(\tau), B(\tau), V(\tau)$ the number of shares of the stock held, the value of bonds held and the market value of the portfolio at time $\tau$. Hence, $V(\tau)=\theta(\tau) P(\tau)+B(\tau), 0 \leq \tau \leq T$.

We seek a self-financing portfolio strategy $\theta(\tau)$ such that the value $V(T)$ of the portfolio is as close as possible to the option's payoff $F(P(T), \sigma(T))$ at $T$. The problem may be written:

$$
\min _{\theta} E\left((V(T)-F(P(T), \sigma(T)))^{2} /\left(V_{0}, P(0), \sigma(0)\right)\right)=\epsilon^{2}\left(V_{0}\right)
$$

The replication error $\epsilon\left(V_{0}\right)$ can be minimized with respect to the initial wealth $V_{0}$ to yield the least-cost optimalreplication strategy and the minimum replication error $\epsilon^{*}$ is $\epsilon^{*}=\min _{V_{0}} \epsilon\left(V_{0}\right)$

In the case of Black and Scholes model, there exists optimal replication strategy for which $\epsilon^{*}=0$, hence "perfect arbitrage" pricing holds.

\footnotetext{
1 LaBAG,Laboratoire Bordelais d'Analyse et Géométrie, UMR 5467, Université Bordeaux1, 33405 Talence Cedex, France
}

(c) EDP Sciences, SMAI 2007 
In the case of dynamically incomplete markets, perfect arbitrage pricing does not hold, for example if the stock price and the volatility are not perfectly correlated; but these markets still admit $\epsilon$-arbitrage strategies for replicating options to within $\epsilon$, where $\epsilon$ can be evaluated numerically.

Consider a stock price process that follows a diffusion process with stochastic volatility:

$$
d P=\mu P d \tau+\sigma P d W_{P}, \quad d \sigma=g(\sigma) d \tau+k \sigma d W_{\sigma}
$$

where $W_{P}$ and $W_{\sigma}$ are Brownian motions with mutual variations $d W_{P} d W_{\sigma}=\rho d \tau$. We define the cost-to-go or value function $J$ by

$$
J(\tau, V, P, \sigma)=\min _{\theta(s), s \geq \tau} E\left((V(T)-F(P(T), \sigma(T)))^{2} /(V(\tau), P(\tau), \sigma(\tau))\right)
$$

Bertsimas, Kogan and Lo have obtained the following results:

a) The value function $J(\tau, V, P, \sigma)$ is quadratic in $V: J=a(V-b)^{2}+c$

b) For $\tau \in[0, T]$, the functions $a, b, c$ satisfy the following system of partial differential equations:

$$
\begin{gathered}
\frac{\partial a}{\partial \tau}=-\frac{k^{2} \sigma^{2}}{2} \frac{\partial^{2} a}{\partial \sigma^{2}}-g_{1}(\sigma) \frac{\partial a}{\partial \sigma}+\rho^{2} k^{2} \frac{\sigma^{2}}{a}\left(\frac{\partial a}{\partial \sigma}\right)^{2}+a f^{2}(\sigma) \\
\frac{\partial b}{\partial \tau}=-\frac{k^{2} \sigma^{2}}{2} \frac{\partial^{2} b}{\partial \sigma^{2}}-\frac{\sigma^{2} P^{2}}{2} \frac{\partial^{2} b}{\partial P^{2}}-\rho k \sigma^{2} P \frac{\partial^{2} b}{\partial \sigma \partial P}-g_{2}(\sigma) \frac{\partial b}{\partial \sigma} \\
-\left(1-\rho^{2}\right) k^{2} \frac{\sigma^{2}}{a} \frac{\partial a}{\partial \sigma} \frac{\partial b}{\partial \sigma} \\
\frac{\partial c}{\partial \tau}=-\frac{k^{2} \sigma^{2}}{2} \frac{\partial^{2} c}{\partial \sigma^{2}}-\frac{\sigma^{2} P^{2}}{2} \frac{\partial^{2} c}{\partial P^{2}}-\rho k \sigma^{2} P \frac{\partial^{2} c}{\partial \sigma \partial P}-g(\sigma) \frac{\partial c}{\partial \sigma}-\sigma f(\sigma) P \frac{\partial c}{\partial P} \\
-\left(1-\rho^{2}\right) k^{2} \sigma^{2} a\left(\frac{\partial b}{\partial \sigma}\right)^{2}
\end{gathered}
$$

where $g(\sigma)=-\delta \sigma\left(\sigma-\sigma_{1}\right)$ with $\delta>0$ and $\left.\sigma_{1} \in\right] 0,1[$,

$$
\begin{aligned}
& g_{1}(\sigma)=g(\sigma)-2 \rho k \sigma f(\sigma), \quad g_{2}(\sigma)=g(\sigma)-\rho k \sigma f(\sigma) \\
& \text { and } f(\sigma)=\left\{\begin{array}{l}
\frac{\mu}{\sigma_{0}} \text { if } \sigma \leq \sigma_{0} \\
\frac{\mu}{\sigma} \text { if } \sigma \geq \sigma_{0}
\end{array}\right.
\end{aligned}
$$

Remark 1.1. The function $f$ has been modified near 0 in order to be bounded and ensure the existence of a solution.

The conditions at the expiry time $T$ are given by: $a(T)=1, b(T)=F(P, \sigma), c(T)=0$.

c) Under the optimal replication strategy $\theta^{*}$, the minimum replication error as a function of the initial wealth $V_{0}$ is $(J(0))^{\frac{1}{2}}=\left(a(0)\left(V_{0}-b(0)\right)^{2}+c(0)\right)^{\frac{1}{2}}$, hence the initial wealth that minimizes the replication error is $V_{0}^{*}=b(0)$ the minimal replication error over all $V_{0}$ is $\epsilon^{*}=\sqrt{c(0)}$; the least-cost optimal strategy at $\tau=0$ is

$$
\theta^{*}(0)=\frac{\partial b}{\partial P}(0)+\frac{\rho k}{P} \frac{\partial b}{\partial \sigma}(0)
$$

Remark 1.2. Exact replication is possible when $k^{2}\left(1-\rho^{2}\right)=0$ and this corresponds to the following cases:

- Volatility is a deterministic function of time.

- The Brownian motions driving stocks prices and volatility are perfectly correlated. 
In this paper, we obtain the existence and uniqueness of solutions of equations (1), (2), (3) in suitable weighted Sobolev spaces such that no boundary condition is needed in 0 and the functions $u_{1}, u_{2}, u_{3}$ have the correct behaviour at infinity, we propose a numerical method to compute the solution and then obtain the minimal replication error and the least-cost optimal replication strategy.

To obtain a forward problem, we change the sense of time; we note $t=T-\tau$. In order to avoid the function $a$ at the denominator, we make the change of unknown $u_{1}=\ln (a)$. We also replace $\sigma$ by $x, P$ by $y, b$ by $u_{2}$ and $c$ by $u_{3}$.

The preceding system becomes:

$$
\begin{gathered}
\frac{\partial u_{1}}{\partial t}-\frac{k^{2} x^{2}}{2} \frac{\partial^{2} u_{1}}{\partial x^{2}}-g_{1}(x) \frac{\partial u_{1}}{\partial x}+k^{2}\left(\rho^{2}-\frac{1}{2}\right) x^{2}\left(\frac{\partial u_{1}}{\partial x}\right)^{2}+f^{2}(x)=0 \\
\frac{\partial u_{2}}{\partial t}-\frac{k^{2} x^{2}}{2} \frac{\partial^{2} u_{2}}{\partial x^{2}}-\frac{x^{2} y^{2}}{2} \frac{\partial^{2} u_{2}}{\partial y^{2}}-\rho k x^{2} y \frac{\partial^{2} u_{2}}{\partial x \partial y}-g_{2}(x) \frac{\partial u_{2}}{\partial x} \\
-\left(1-\rho^{2}\right) k^{2} x^{2} \frac{\partial u_{1}}{\partial x} \frac{\partial u_{2}}{\partial x}=0 \\
\frac{\partial u_{3}}{\partial t}-\frac{k^{2} x^{2}}{2} \frac{\partial^{2} u_{3}}{\partial x^{2}}-\frac{x^{2} y^{2}}{2} \frac{\partial^{2} u_{3}}{\partial y^{2}}-\rho k x^{2} y \frac{\partial^{2} u_{3}}{\partial x \partial y}-g(x) \frac{\partial u_{3}}{\partial x}-x f(x) y \frac{\partial u_{3}}{\partial y} \\
-\left(1-\rho^{2}\right) k^{2} x^{2} \exp \left(u_{1}\right)\left(\frac{\partial u_{2}}{\partial x}\right)^{2}=0
\end{gathered}
$$

with the initial conditions:

$$
u_{1}(0)=0, \quad u_{2}(0)=F(x, y), \quad u_{3}(0)=0,
$$

where $F$ is the payoff function.

\section{Computation of $u_{1}$}

To simplify the notations, we denote:

$$
F_{1}(x)=f^{2}(x), x>0, \quad \lambda=k^{2}\left(\rho^{2}-\frac{1}{2}\right)
$$

The coefficient $\lambda$ may be positive or negative since $\rho$ is a correlation factor and then $\rho$ lies in $[-1,+1]$.

The equation (4) becomes:

$$
\frac{\partial u_{1}}{\partial t}-\frac{1}{2} k^{2} x^{2} \frac{\partial^{2} u_{1}}{\partial x^{2}}-g_{1}(x) \frac{\partial u_{1}}{\partial x}+\lambda x^{2}\left(\frac{\partial u_{1}}{\partial x}\right)^{2}+F_{1}(x)=0
$$

We will make the following assumptions on the functions $f$ and $g_{1}$ :

- $f \in W^{1, \infty}\left(\mathbb{R}^{+}\right), x f^{\prime} \in L^{\infty}\left(\mathbb{R}^{+}\right), x f^{\prime \prime} \in L^{\infty}\left(\mathbb{R}^{+}\right)$.

- $F_{1}$ is a nonincreasing function, $F_{1}^{\prime \prime} \in L^{1}\left(\mathbb{R}^{+}\right)$; if we denote $\hat{F}$ the function defined by $\hat{F}(x)=$ $x F_{1}^{\prime}(x), x>0$, we get $\hat{F} \in L^{\infty}\left(\mathbb{R}^{+}\right)$.

- $g_{1}$ may be written $g_{1}(x)=x \phi(x)$ with $\phi(x)=-\delta\left(x-\sigma_{1}\right)-2 \rho k f(x), \delta>0$; so, there exists $\sigma_{2}>0$ such that $\phi$ is negative and nonincreasing on $\left[\sigma_{2},+\infty\left[\right.\right.$ and bounded on $\left[0, \sigma_{2}\right]$.

We denote by $\Delta t_{n}$ the time increment between the levels $t_{n}$ and $t_{n+1}, n \geq 0$ and by $u_{1 h}^{n}$ the approximate solution at the time level $t_{n}$. This solution will be in a finite-dimensional space $V_{1 h}$ which will be defined below. 
The solution $u_{1 h}^{n+1}$ at the time level $t_{n+1}$ is computed in two steps: knowing $u_{1 h}^{n}$, we compute $u_{1 h}^{n+\frac{1}{2}}$, approximate solution of

$$
\frac{\partial u_{1}}{\partial t}+\lambda x^{2}\left(\frac{\partial u_{1}}{\partial x}\right)^{2}=0
$$

obtained by using an explicit upwind scheme. Then starting with this intermediate value, we use a backward Euler method in time to compute $u_{1 h}^{n+1}$; the second order term in (7) is discretized by using a $P_{1}$-finite element method and the linear first order term by an implicit upwind scheme in order to get the $L^{\infty}$-stability of the scheme.

In order to solve the parabolic problem:

$$
\frac{\partial u_{1}}{\partial t}-\frac{1}{2} k^{2} x^{2} \frac{\partial^{2} u_{1}}{\partial x^{2}}+F_{1}(x)=0
$$

we introduce the Sobolev space:

$$
V_{1}=\left\{v \in \mathcal{D}^{\prime}\left(\mathbb{R}^{+}\right) / \frac{v}{1+x} \in L^{2}\left(\mathbb{R}^{+}\right), \frac{x v^{\prime}}{1+x} \in L^{2}\left(\mathbb{R}^{+}\right)\right\}
$$

The space $V_{1}$ with the norm

$$
\|v\|_{V_{1}}=\left(\int_{0}^{\infty}\left(\frac{v^{2}(x)}{(1+x)^{2}}+\frac{x^{2}}{(1+x)^{2}}\left(\frac{d v}{d x}\right)^{2}\right) d x\right)^{\frac{1}{2}}
$$

is a Hilbert space and $\mathcal{D}\left(\mathbb{R}^{+}\right)$is dense in $V_{1}[3]$.

We define the bilinear form on $V_{1} \times V_{1}$

$$
\forall v, w \in V_{1}, a(v, w)=\frac{1}{2} k^{2} \int_{0}^{+\infty} \frac{d v}{d x} \frac{d}{d x}\left(w \frac{x^{2}}{(1+x)^{2}}\right) d x
$$

This bilinear form is continuous on $V_{1} \times V_{1}$ and satisfies the Gårding's inequality; hence, the following problem:

Find $u_{1} \in L^{2}\left(0, T ; V_{1}\right) \cap C\left(0, T ; H_{1}\right)$ such that:

$$
\left\{\begin{aligned}
\left(\frac{\partial u_{1}}{\partial t}, v\right)_{1}+a\left(u_{1}, v\right) & =-\left(F_{1}, v\right)_{1}, \forall v \in V_{1} \\
u_{1}(0) & =0
\end{aligned}\right.
$$

has a unique solution.

The finite-dimensional space $V_{1 h}$ will be a subspace of $V_{1}$ defined in the following way:

Let $\left(x_{i}\right)_{0 \leq i \leq N}$ an increasing sequence $\left(x_{0}=0\right)$. We denote $h_{i}=x_{i}-x_{i-1}, I_{i}=\left(x_{i-1}, x_{i}\right), 1 \leq i \leq N$, $I_{N+1}=\left(x_{N},+\infty\right)$

$$
V_{1 h}=\left\{v_{h} \in C^{0}\left(\mathbb{R}^{+}\right) / v_{h \mid I_{i}} \in P_{1}, 1 \leq i \leq N, v_{h \mid I_{N+1}} \in P_{0}\right\}
$$

The variable $x$ is the volatility which lies, in practice, in $] 0,1[$, so, we may use a constant space step $h$ on $(0,1)$ and an increasing sequence $\left(h_{i}\right)$ for $x \geq 1$ in order that the number of nodes is not too large.

If $v_{h} \in V_{1 h}$, we denote $v_{i}=v_{h}\left(x_{i}\right)$.

The approximate solution of (9) at the time level $t_{n+1}$ is the solution of:

$$
\left(u_{1 h}^{n+1}, v_{h}\right)_{h}+\Delta t_{n} a\left(u_{1 h}^{n+1}, v_{h}\right)=\left(u_{1 h}^{n}, v_{h}\right)_{h}-\Delta t_{n}\left(\pi_{h} F_{1}, v_{h}\right)_{h}, \quad \forall v_{h} \in V_{1 h}
$$


where $\left(u_{h}, v_{h}\right)_{h}$ is an approximate scalar product and $\pi_{h} F_{1}$ the Lagrange interpolate of $F_{1}$.

We compute now an approximate solution of (8) by using an explicit upwind scheme.

Let us denote by $v_{h}^{n}$ the derivative of $u_{1 h}^{n}$

$$
v_{h \mid I_{i}}^{n}=v_{i}^{n}=\frac{u_{1 i}^{n}-u_{1, i-1}^{n}}{h_{i}}, \quad 1 \leq i \leq N, v_{h \mid I_{N+1}}=v_{N+1}^{n}=0
$$

We set $v_{0}^{n}=0$.

It is proved that the function $v_{h}^{n}$ is positive and the function $u_{1 h}^{n}$ is negative; we define $u_{1 h}^{n+\frac{1}{2}} \in V_{1 h}$ by:

$$
\begin{gathered}
u_{1 i}^{n+\frac{1}{2}}=u_{1 i}^{n}-\lambda \Delta t_{n} x_{i}^{2}\left(v_{i}^{n}\right)^{2} \text { if } \lambda>0 \\
u_{1 i}^{n+\frac{1}{2}}=u_{1 i}^{n}-\lambda \Delta t_{n} x_{i+1}^{2}\left(v_{i+1}^{n}\right)^{2} \text { if } \lambda<0
\end{gathered}
$$

$0 \leq i \leq N$

For the linear first order term, since the function $g_{1}$ is not bounded, we used an implicit scheme, which will be decentered in order to get a monotone matrix.

Finally, the solution $u_{1 h}^{n+1} \in V_{1 h}$ of $(7)$ is defined by:

$$
\begin{gathered}
u_{10}^{n+1}=u_{10}^{n+\frac{1}{2}}-\Delta t_{n} F_{10} \\
u_{1 i}^{n+1}+\Delta t_{n}\left(\alpha_{i}\left(\frac{1}{h_{i}}+\frac{1}{h_{i+1}}\right)+\gamma_{i}\left(\frac{1-\delta_{i}}{h_{i+1}}-\frac{\delta_{i}}{h_{i}}\right)\right) u_{1 i}^{n+1} \\
-\frac{\Delta t_{n}}{h_{i}}\left(\alpha_{i}-\gamma_{i} \delta i\right) u_{1, i-1}^{n+1}-\frac{\Delta t_{n}}{h_{i+1}}\left(\alpha_{i}+\gamma_{i}\left(1-\delta_{i}\right)\right) u_{1, i+1}^{n+1} \\
=u_{1 i}^{n+\frac{1}{2}}-\Delta t_{n} F_{1 i}, 1 \leq i \leq N-1 \\
u_{1 N}^{n+1}+\frac{\Delta t_{n}}{h_{N}}\left(\alpha_{N}-\gamma_{N}\right)\left(u_{1 N}^{n+1}-u_{1, N-1}^{n+1}\right)=u_{1 N}^{n+\frac{1}{2}}-\Delta t_{n} F_{1 N}
\end{gathered}
$$

with

$$
\begin{aligned}
& \alpha_{i}=\frac{k^{2} x_{i}^{2}}{h_{i}+h_{i+1}}, 1 \leq i \leq N-1, \alpha_{N}=\frac{k^{2} x_{N}^{2}}{h_{N}+2\left(1+x_{N}\right)}, \\
& \gamma_{i}=g_{1}\left(x_{i}\right), 1 \leq i \leq N, \\
& \delta_{i}=0 \text { if } \gamma_{i}>0 \text { and } \delta_{i}=1 \text { if } \gamma_{i} \leq 0 .
\end{aligned}
$$

Under a stability condition, we obtain estimates of the approximate solution:

Proposition 2.1. If the following stability condition is satisfied:

$$
\sup _{i \geq 1}\left|\lambda \frac{\Delta t_{n}}{h_{i}} x_{i}^{2} v_{i}^{n}\right| \leq 1
$$

then the function $v_{h}^{n}$ is nonnegative and the function $u_{h}^{n}$ is nonpositive for $n \geq 0$. Besides, the following estimates hold:

$$
\left\|u_{1 h}^{n}\right\|_{L^{\infty}\left(\mathbb{R}^{+}\right)} \leq t_{n} F_{1}(0), \quad\left\|v_{h}^{n}\right\|_{L^{1}\left(\mathbb{R}^{+}\right)} \leq t_{n} F_{1}(0)
$$


It is possible to choose the nodes $\left(x_{i}\right), 1 \leq i \leq N$ such that the stability condition (14) is not too restrictive. We get in the following proposition that under some hypothesis on the sequence $\left(h_{i}\right)$ the function $x v_{h}^{n}$ is bounded in $L^{\infty}\left(\mathrm{R}^{+}\right)$.

We define the function $\hat{v}_{h}^{n}$ by:

$$
\hat{v}_{h \mid I_{i}}^{n}=\hat{v}_{i}^{n}=x_{i} v_{i}^{n}, 1 \leq i \leq N, \hat{v}_{h \mid I_{N+1}}^{n}=\hat{v}_{N+1}^{n}=0 .
$$

Proposition 2.2. If the following stability condition

$$
\sup _{i \geq 1} \lambda \frac{\Delta t_{n}}{h_{i}} x_{i}\left(\hat{v}_{i}^{n}+\hat{v}_{i-1}^{n}\right) \leq 1 \text { if } \lambda>0 \quad \text { and } \sup _{i \geq 1}|\lambda| \frac{\Delta t_{n}}{h_{i}} x_{i}\left(\hat{v}_{i}^{n}+\hat{v}_{i+1}^{n}\right) \leq 1 \text { if } \lambda<0
$$

is satisfied and if the sequence $\left(h_{i}\right)_{1 \leq i \leq N}$ satisfy: There exists a positive constant c such that

$$
\frac{x_{i}}{x_{i+1}} \frac{h_{i+1}}{h_{i}+h_{i+1}}-\frac{x_{i-1}}{x_{i}} \frac{h_{i}}{h_{i}+h_{i-1}} \geq-c \frac{h_{i}}{x_{i}}, 1 \leq i \leq N-1
$$

and

$$
\frac{x_{N}}{h_{N}+2\left(1+x_{N}\right)}-\frac{x_{N-1}}{x_{N}} \frac{h_{N}}{h_{N}+h_{N-1}} \geq-c \frac{h_{N}}{x_{N}},
$$

then if $\Delta t \leq \Delta t_{0}, \Delta t_{0}$ depending on $c, c_{1}, c_{2}$, the following estimate holds:

$$
\left\|\hat{v}_{h}^{n}\right\|_{L^{\infty}\left(\mathbb{R}^{+}\right)} \leq e^{C t_{n}}\|\hat{F}\|_{L^{\infty}\left(\mathbb{R}^{+}\right)}
$$

for $n \geq 0$ and $C$ is a constant depending on $c, c_{1}, c_{2}$.

If we define the sequence $\left(x_{i}\right)$ by $x_{i}=i h, 0 \leq i \leq n_{0}$ with $n_{0} h=1$, and $x_{i}=e^{\theta h} x_{i-1}$ for $i \geq n_{0}+1$ and $\theta \geq 1$, then the inequalities (16) and (17) are satisfied if $N=\mathcal{O}\left(\frac{|\ln h|}{h}\right)$ or $x_{N}=\mathcal{O}\left(\frac{1}{h}\right)$.

Besides we have $\frac{x_{i}}{h_{i}}=\mathcal{O}\left(\frac{1}{h}\right) 1 \leq i \leq N$, and the stability condition may be written $\frac{\Delta t_{n}}{h} \leq C$, that is the classical stability condition for hyperbolic problem.

From all these estimates, we can deduce the convergence of the numerical solution to a weak solution of (7). Besides, this solution is unique. Therefore, this problem admits a unique weak solution.

Fig 1 represents $a(0)$ with a time maturity equal to 1 for different values of $\rho ; \sigma_{0}=0.01$ and the values of the other parameters are those proposed in [1]: $k=0.4, \delta=2, \sigma_{1}=0.153, \mu=0.7$.

\section{Computation of $u_{2}$}

$u_{2}$ is solution of

$$
\begin{gathered}
\frac{\partial u_{2}}{\partial t}-\frac{k^{2} x^{2}}{2} \frac{\partial^{2} u_{2}}{\partial x^{2}}-\frac{x^{2} y^{2}}{2} \frac{\partial^{2} u_{2}}{\partial y^{2}}-\rho k x^{2} y \frac{\partial^{2} u_{2}}{\partial x \partial y}-g_{2}(x) \frac{\partial u_{2}}{\partial x} \\
-\left(1-\rho^{2}\right) k^{2} x^{2} \frac{\partial u_{1}}{\partial x} \frac{\partial u_{2}}{\partial x}=0
\end{gathered}
$$

The initial condition is the payoff function. If the European option is a put, the payoff function is given by: $F(y)=\operatorname{Max}(E-y, 0)$ if $E$ is the exercise price. 


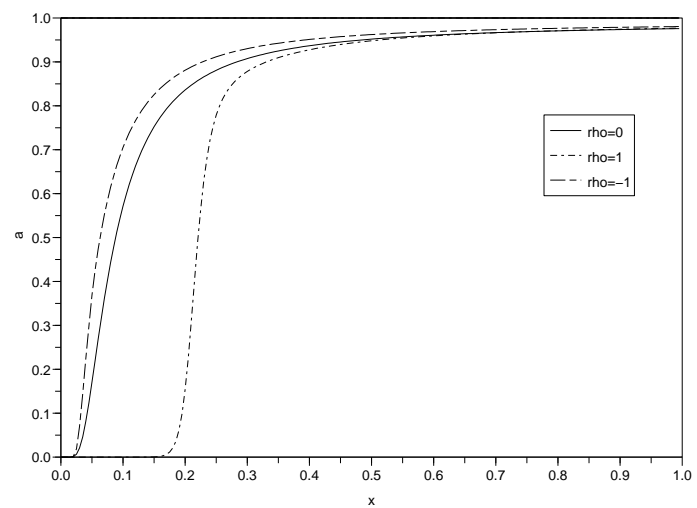

Figure 1. $a(0)$ with maturity time $T=1$

In order to obtain a bilinear form satisfying Gårding's inequality, we make a change of unknown. We denote: $\hat{u}_{2}=e^{-\alpha x} u_{2}, \alpha>0$. The function $\hat{u}_{2}$ is solution of:

$$
\begin{gathered}
\frac{\partial \hat{u}_{2}}{\partial t}-\frac{k^{2}}{2} x^{2} \frac{\partial^{2} \hat{u}_{2}}{\partial x^{2}}-\frac{x^{2} y^{2}}{2} \frac{\partial^{2} \hat{u}_{2}}{\partial y^{2}}-\rho k x^{2} y \frac{\partial^{2} \hat{u}_{2}}{\partial x \partial y}-\frac{\partial \hat{u}_{2}}{\partial x}\left(\alpha k^{2} x^{2}+g_{2}(x)+\left(1-\rho^{2}\right) k^{2} x^{2} \frac{\partial u_{1}}{\partial x}\right) \\
-\rho \alpha k x^{2} y \frac{\partial \hat{u}_{2}}{\partial y}-\left(\frac{\alpha^{2} k^{2}}{2} x^{2}+\alpha g_{2}(x)+\left(1-\rho^{2}\right) \alpha k^{2} x^{2} \frac{\partial u_{1}}{\partial x}\right) \hat{u}_{2}=0
\end{gathered}
$$

with the initial condition: $\hat{u}_{2}(0)=e^{-\alpha x} F(y)$.

We define a variational formulation of this problem. Let us consider the following space $\hat{V}_{2}$ defined by:

$$
\hat{V}_{2}=\left\{v \in \mathcal{D}^{\prime}(\Omega) / v \in L^{2}(\Omega), x v \in L^{2}(\Omega), x v_{x} \in L^{2}(\Omega), x y v_{y} \in L^{2}(\Omega)\right\}
$$

with $\Omega=\mathbb{R}^{+} \times \mathbb{R}^{+}$.

This space with the norm:

$$
\|v\|=\left(\int_{\Omega} v^{2}+x^{2} v^{2}+x^{2}\left(\frac{\partial v}{\partial x}\right)^{2}+x^{2} y^{2}\left(\frac{\partial v}{\partial y}\right)^{2}\right)^{\frac{1}{2}}
$$

is a Hilbert space and $\mathcal{D}(\Omega)$ is dense in $\hat{V}_{2}[3]$.

Besides, we have the estimates:

$$
\|x v\|_{L^{2}(\Omega)} \leq 2\left\|x y \frac{\partial v}{\partial y}\right\|_{L^{2}(\Omega)}, \quad\|v\|_{L^{2}(\Omega)} \leq 2\left\|x \frac{\partial v}{\partial x}\right\|_{L^{2}(\Omega)}
$$

and the semi-norm:

$$
\|v\|_{2}=\left(\left\|x \frac{\partial v}{\partial x}\right\|_{L^{2}(\Omega)}^{2}+\left\|x y \frac{\partial v}{\partial y}\right\|_{L^{2}(\Omega)}^{2}\right)^{2}
$$

is a norm in $\hat{V}_{2}$ equivalent to the norm $\|\cdot\|_{\hat{V}_{2}}$. 


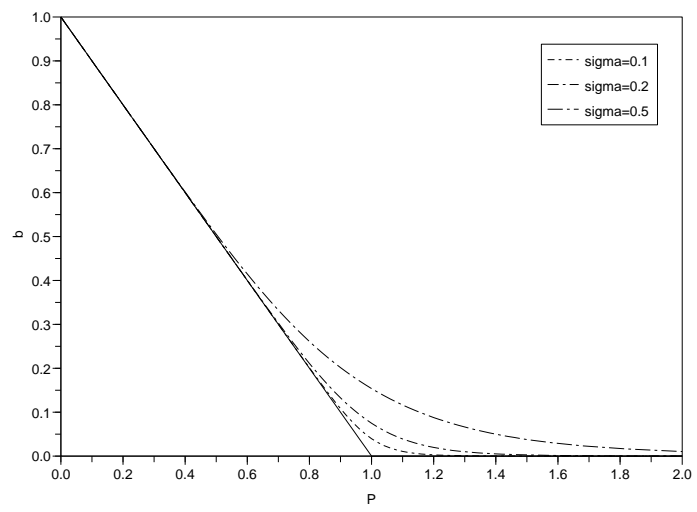

FiguRE 2. $b(0)$ with maturity time $T=1$

We define on $\hat{V}_{2} \times \hat{V}_{2}$ the bilinear form $\hat{b}$ by:

$$
\begin{aligned}
\forall u, v \in \hat{V}_{2}, \quad \hat{b}(u, v)= & \frac{k^{2}}{2} \int_{\Omega} \frac{\partial u}{\partial x} \frac{\partial}{\partial x}\left(x^{2} v\right) d x d y+\frac{1}{2} \int_{\Omega} x^{2} \frac{\partial u}{\partial y} \frac{\partial}{\partial y}\left(y^{2} v\right) d x d y \\
& +\rho k \int_{\Omega} x^{2} \frac{\partial u}{\partial x} \frac{\partial}{\partial y}(y v) d x d y-\int_{\Omega}\left(\alpha k^{2} x^{2}+g_{2}(x)+\left(1-\rho^{2}\right) k^{2} x^{2} \frac{\partial u_{1}}{\partial x}\right) \frac{\partial u}{\partial x} v d x d y \\
& -\rho \alpha k \int_{\Omega} x^{2} y \frac{\partial u}{\partial y} v d x d y-\int_{\Omega}\left(\frac{\alpha^{2} k^{2}}{2} x^{2}+\alpha g_{2}(x)+\left(1-\rho^{2}\right) \alpha k^{2} x^{2} \frac{\partial u_{1}}{\partial x}\right) u v d x d y
\end{aligned}
$$

Proposition 3.1. If $|\rho|<1$ and $k<\frac{2}{3} \delta$, there exists positive constants $C, c, \alpha$ depending on $\delta, \sigma_{1}, k, \rho$ such that

$$
\forall v \in \hat{V}_{2}, \hat{b}(v, v) \geq C\|v\|_{2}^{2}-c\|v\|_{L^{2}(\Omega)}^{2} .
$$

We may write problem (19) in variational form:

$$
\left\{\begin{array}{c}
\text { Find } \hat{u}_{2} \in C\left(0, T ; L^{2}(\Omega)\right) \cap L^{2}\left(0, T ; \hat{V}_{2}\right) \\
\left(\frac{\partial \hat{u}_{2}}{\partial t}, v\right)+\hat{b}\left(\hat{u}_{2}, v\right)=0, \forall v \in V_{2}, \\
\hat{u}_{2}(0)=e^{-\alpha x} F
\end{array}\right.
$$

It results from Proposition 3.1 that this problem admits a unique solution.

The problem is solved by using a backward Euler method in time and a suitable finite element method in space.

In Fig 2, we present the variation of $u_{2}(T)$ in $y$ (or $b(0)$ in $P$ ) for different values of the volatility; the parameter $\rho$ is null; the exercise price $E$ is equal to 1 ; the other parameters have the same values as in Fig 1.

\section{Computation of $u_{3}$}

$u_{3}$ is solution of

$$
\frac{\partial u_{3}}{\partial t}-\frac{k^{2} x^{2}}{2} \frac{\partial^{2} u_{3}}{\partial x^{2}}-\frac{x^{2} y^{2}}{2} \frac{\partial^{2} u_{3}}{\partial y^{2}}-\rho k x^{2} y \frac{\partial^{2} u_{3}}{\partial x \partial y}-g(x) \frac{\partial u_{3}}{\partial x}-x f(x) y \frac{\partial u_{3}}{\partial y}=\left(1-\rho^{2}\right) k^{2} x^{2} \exp \left(u_{1}\right)\left(\frac{\partial u_{2}}{\partial x}\right)^{2}
$$




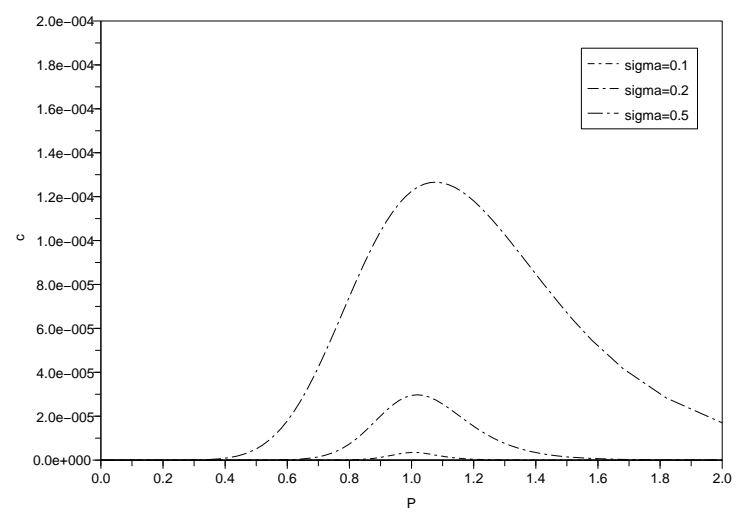

FiguRE 3. $c(0)$ with maturity time $T=1$

with the initial condition: $u_{3}(0)=0$.

As for the computation of $u_{2}$, we make the change of unknown: $\hat{u}_{3}=e^{-\alpha x} u_{3}$ and $\hat{u}_{3}$ is solution of:

$$
\begin{gathered}
\frac{\partial \hat{u}_{3}}{\partial t}-\frac{k^{2} x^{2}}{2} \frac{\partial^{2} \hat{u}_{3}}{\partial x^{2}}-\frac{x^{2} y^{2}}{2} \frac{\partial^{2} \hat{u}_{3}}{\partial y^{2}}-\rho k x^{2} y \frac{\partial^{2} \hat{u}_{3}}{\partial x \partial y}-\frac{\partial \hat{u}_{3}}{\partial x}\left(\alpha k^{2} x^{2}+g(x)\right)-\frac{\partial \hat{u}_{3}}{\partial y}\left(\rho k \alpha x^{2} y+x f(x) y\right) \\
-\hat{u}_{3}\left(\frac{\alpha^{2} k^{2} x^{2}}{2}+\alpha g(x)\right)=\left(1-\rho^{2}\right) k^{2} x^{2} \exp \left(u_{1}-\alpha x\right)\left(\frac{\partial u_{2}}{\partial x}\right)^{2}
\end{gathered}
$$

In a similar way as for $u_{2}$, we obtain the existence and uniqueness of a solution of (21) in $\hat{V}_{2}$ and we use the same numerical method to compute $u_{3}$.

Fig 3 represents the variation of $u_{3}(T)$ in $y$ (or $c(0)$ in $P$ ) for different values of $\sigma$. The parameter $\rho$ is equal to 0 . (In the case $\rho=1$ or $\rho=-1, u_{3}$ is zero). The replication error $\epsilon^{*}$ is given by: $\epsilon^{*}=\sqrt{c(0)}$.

Knowing $u_{2}$, we can compute the least-cost optimal strategy

$$
\theta^{*}(0)=\frac{\partial b}{\partial P}(0)+\frac{\rho k}{P} \frac{\partial b}{\partial \sigma}(0)=\frac{\partial u_{2}}{\partial y}(T)+\frac{\rho k}{y} \frac{\partial u_{2}}{\partial y}(T)
$$

Fig 4 represents the variation of $\theta^{*}(0)$ in $P$ for different values of $\sigma$; the parameter $\rho$ is equal to 0 .

\section{REFERENCES}

[1] Bertsimas D., Kogan L., and Lo A.W. Hedging derivative securities and incomplete markets: An $\epsilon$-arbitrage approach. Operations Research, 49(3):372-397, 2001.

[2] Black F. and Scholes M. Pricing of options and corporate liabilities. J. of Political Econom., 81:637-654, 1973.

[3] Bolley P. and Camus J. Quelques résultats sur les espaces de Sobolev avec poids. Publications de Séminaires Mathématiques de Rennes, 1:1-69, 1969 


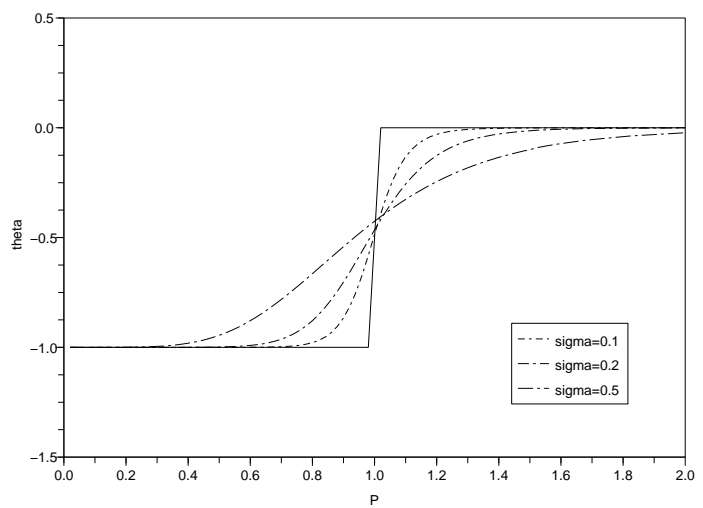

Figure 4 . Optimal strategy with maturity time $T=1$ 